\title{
Multiwavelength Modeling the SED of Strongly Interacting Binaries
}

\author{
Augustin Skopal \\ Astronomical Institute of the Slovak Academy of Sciences, \\ 05960 Tatranská Lomnica, Slovakia \\ email: skopal@ta3.sk
}

\begin{abstract}
The spectrum of strongly interacting binaries, as for example, high and low mass $\mathrm{X}$-ray binaries, symbiotic (X-ray) binaries and/or classical and recurrent novae, consists of more components of radiation contributing from hard X-rays to radio wavelengths. To understand the basic physical processes responsible for the observed spectrum we have to disentangle the composite spectrum into its individual components, i.e. to determine their physical parameters. In this short contribution I demonstrate the method of modeling the multiwavelength SED on the example of the extragalactic super-soft X-ray source RX J0059.1-7505 (LIN 358).
\end{abstract}

Keywords. stars: fundamental parameters, X-rays: individual (RX J0059.1-7505)

\section{Introduction}

Strongly interacting binaries consist of an accreting compact object and a low-mass $\left(\leqslant 1 M_{\odot}\right)$ main-sequence or slightly evolved late-type star. In special cases, the donor star can be an M-type giant. The mass transfer from the cool component to the compact one, represents principal interaction in these binaries. It can happen via the Roche lobe overflow in short-period binaries, e.g. cataclysmic variables, or via the wind in extended systems as, e.g., symbiotic stars. These objects are subject to occasional outbursts, during which a large amount of radiative and kinetic energy is liberated. A part of the kinetic energy of particles can be converted to radiation due to shocked gas that creates an extremely hot plasma. Its radiation can be detected at hard to soft X-rays. The hot star radiation from, e.g., a burning white dwarf, can be measured directly at the supersoft X-rays and the far-UV. A fraction of its radiation is re-processed via the ionization/recombination acts into the nebular radiation, which signatures are best indicated at the near-UV and the radio. The contribution from the giant dominates usually the optical/near-IR. In some cases, the post-outburst circumstellar material or that produced by a mira-type giant can condense in dust particles, which can re-process the radiation from the binary components into a dust emission, seen in the infrared domain. As a result, the observed spectrum of strongly interacting binaries is composed of different components of radiation. Figure 1 shows an example of the above described components. Their extraction from the composite spectrum can aid us in understanding physical processes giving rise the observed result. Here, I present the observed and model SED for the extragalactic super-soft X-ray sources, RX J0059.1-7505. The method of disentangling the composite spectrum was described by Skopal et al. (2009).

\section{Multiwavelength model SED of RX J0059.1-7505}

This star is classified as a symbiotic X-ray binary. Figure 2 shows its observed and model SED from the supersoft X-rays to the near-IR. The X-ray fluxes were reconstructed 


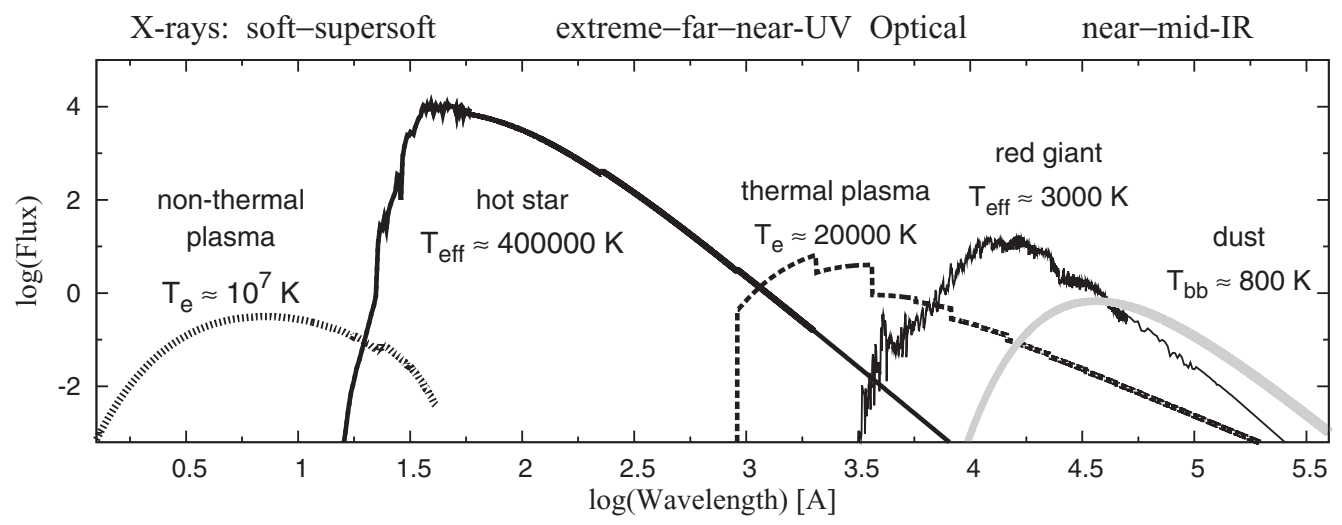

Figure 1. Scheme of main components of radiation in the continuum that can contribute to the X-ray to mid-IR spectrum of strongly interacting binaries.

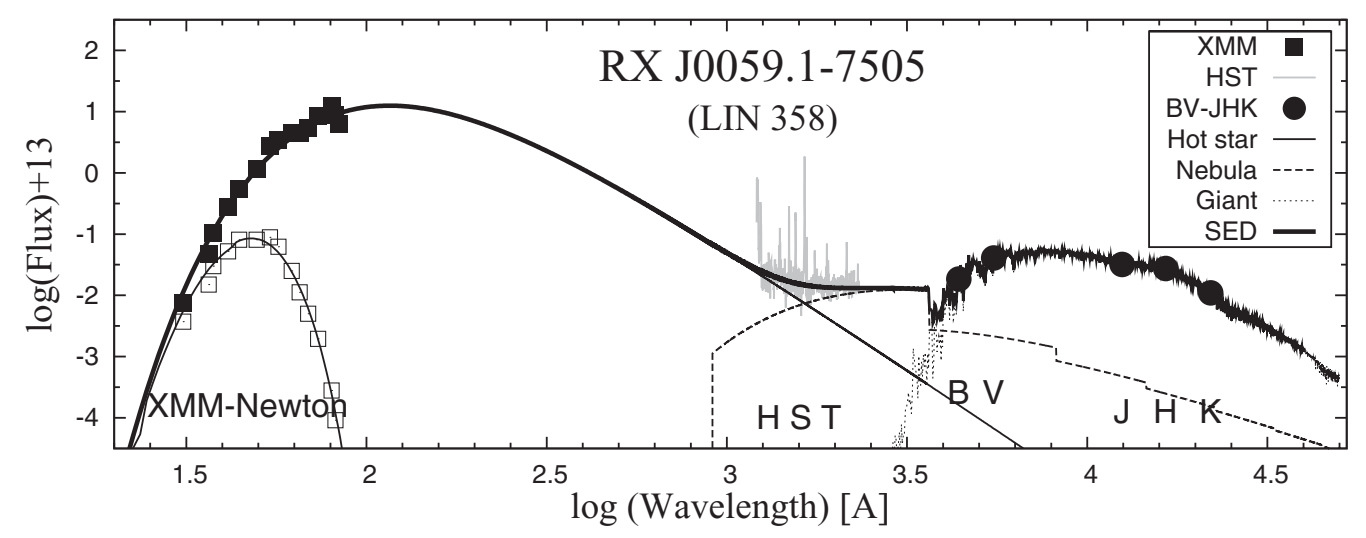

Figure 2. A comparison of the measured (in keys) and modeled (solid heavy line) SED of the symbiotic X-ray binary in the Small Magellanic Cloud, RX J0059.1-7505 (LIN 358). Open/filled squares are the absorbed/unabsorbed X-ray fluxes. Fluxes are in units of $\operatorname{erg~cm}^{-2} \mathrm{~s}^{-1} \AA^{-1}$.

from Fig. 4 of Kahabka \& Haberl (2006). The HST ultraviolet spectra were obtained from the satellite archive, and the $B V J H K$ photometric measurements were published by Mürset et al. (1996). The preliminary model SED corresponds to the hot star parameters, $T_{\mathrm{h}}=250000 \mathrm{~K}, R_{\mathrm{h}}=0.09 \mathrm{R}_{\odot}$ and $L_{\mathrm{h}}=1.1 \times 10^{38} \mathrm{erg} \mathrm{s}^{-1}$, which radiation is attenuated with $N_{\mathrm{H}}=6.1 \times 10^{20} \mathrm{~cm}^{-2}$. The nebula has $T_{\mathrm{e}}=18000 \mathrm{~K}$ and its amount corresponds to the emission measure, $E M=2.4 \times 10^{60} \mathrm{~cm}^{-3}$. The giant in LIN 358 can be classified as a K5 Ib supergiant, because its $T_{\text {eff }}=4000 \pm 200 \mathrm{~K}, R_{\mathrm{g}}=178(d / 60 \mathrm{kpc}) \mathrm{R}_{\odot}$ and $L_{\mathrm{g}} \sim 7300(d / 60 \mathrm{kpc})^{2} \mathrm{~L}_{\odot}$, as given by the model SED.

\section{Acknowledgement}

This research was supported by a grant of the SAS, VEGA No. 2/0038/10.

\section{References}

Kahabka, P. \& Haberl, F. 2006, A\& A, 452, 431

Mürset, U., Schild, H., \& Vogel, M. 1996, A\&A, 307, 516

Skopal, A., Sekeráš, M., Gonzĺez-Riestra, R., \& Viotti, R. F. 2009, A\&A, 507, 1531 